\title{
Errors in visuo-haptic and haptic-haptic location matching are stable over long periods of time
}

\author{
Irene A. Kuling *, Eli Brenner, Jeroen B.J. Smeets \\ MOVE Research Institute Amsterdam, Department of Human Movement Sciences, VU University, Amsterdam, Netherlands
}

\section{A R T I C L E I N F O}

\section{Article history:}

Received 3 July 2015

Received in revised form 8 February 2016

Accepted 22 March 2016

Available online $\mathrm{xxxx}$

\section{Keywords:}

Visuo-haptic biases

Drift

Proprioception

Hand localization

\begin{abstract}
A B S T R A C T
People make systematic errors when they move their unseen dominant hand to a visual target (visuo-haptic matching) or to their other unseen hand (haptic-haptic matching). Why they make such errors is still unknown. A key question in determining the reason is to what extent individual participants' errors are stable over time. To examine this, we developed a method to quantify the consistency. With this method, we studied the stability of systematic matching errors across time intervals of at least a month. Within this time period, individual subjects' matches were as consistent as one could expect on the basis of the variability in the individual participants' performance within each session. Thus individual participants make quite different systematic errors, but in similar circumstances they make the same errors across long periods of time.
\end{abstract}

(C) 2016 Elsevier B.V. All rights reserved.

\section{Introduction}

When reaching for a visual object with an unseen hand people make both variable and systematic errors. Variable errors presumably arise from variability in sensory processing, movement planning and movement execution (van Beers, 2009). It is less clear why people make systematic errors, and continue to do so after feedback has been provided (Smeets, van den Dobbelsteen, de Grave, van Beers, \& Brenner, 2006). Some systematic errors, such as direction-dependent distortions in force magnitude perception (van Beek, Tiest, \& Kappers, 2013) or in distance perception (radial-tangential illusion; (Wong, 1977), could be explained by general characteristics of the biomechanics of the arm. However, many systematic errors that have been found in reaching for visual targets with an unseen hand cannot be explained by general human characteristics, because they differ considerably between subjects: they are idiosyncratic.

Several studies have examined the errors that are found when matching a visually perceived with a haptically perceived position (visuo-haptic task; (Van Beers, Sittig, \& Denier van der Gon, 1998; Smeets et al., 2006; Sousa, Brenner, \& Smeets, 2010; Rincon-Gonzalez, Buneo, \& Helms Tillery, 2011; Kuling, Brenner, \& Smeets, 2013; Van der Kooij, Brenner, van Beers, Schot, \& Smeets, 2013; Kuling, van der Graaff, Brenner, \& Smeets, 2014; Kuling, Brenner, \& Smeets, 2015). The matching errors are typically smaller for targets closer to the body (Van Beers et al., 1998), and they are not influenced by external forces (Kuling et al., 2013, 2015). Substantial matching errors have also been

\footnotetext{
* Corresponding author.

E-mail address: i.a.kuling@vu.nl (I.A. Kuling).
}

found in tasks in which the position of one of the hands has to be matched with the other hand (haptic-haptic tasks; (Von Hofsten \& Rösblad, 1988; Haggard, Newman, Blundell, \& Andrew, 2000).

In order to perform the matching task, sensory input about the target position must be transformed into motor commands that will bring the hand to the target. As the hand approaches and reaches the target, any felt mismatch between the position of the moving hand and that of the target or of the other hand can be used to adjust the movement itself or subsequent movements. Consequently, both errors specific for a sensory modality (Smeets et al., 2006; Bernier, Gauthier, \& Blouin, 2007) and errors in the transformation between vision and haptics (McIntyre \& Lipshits, 2008) might contribute to visuo-haptic matching errors.

The results of several studies suggest that matching errors are stable over time (Smeets et al., 2006; Rincon-Gonzalez et al., 2011). RinconGonzalez et al. (2011) specifically included time as a factor when studying visuo-haptic matching errors. In their study, the subject's right arm was moved passively (by the experimenter) towards a location, while the subject had his eyes closed. The subject had to remember the position of the hand. The hand was then moved back to a start position, and the subject opened his eyes and indicated the reached position on a grid by naming the coordinate of the position. Four months later they re-recruited the same subjects to do the same experiment with the left hand. Their subjects showed very similar error patterns in the two experiments, so the authors concluded that the proprioceptive map is stable across hands and time. Smeets et al. (2006) looked at visuo-haptic matching errors in a task in which subjects had to reach to 3D virtual targets. They found that the subjects' visuo-haptic matching errors were very similar between two blocks in a single session and between sessions on different days. 
We also found evidence for stable visuo-haptic and haptic-haptic matching errors in our previous experiments in which participants had to move their hand to indicated positions (Kuling et al., 2013, 2014, submitted for publication). We studied several force manipulations (Kuling et al., 2013, 2015) and skin stretch manipulations (Kuling et al., submitted for publication) in tasks that involved repeated sessions, and found idiosyncratic visuo-haptic matching errors on different blocks and sessions within each experiment.

The above-mentioned studies suggest that visuo-haptic and haptichaptic matching errors are quite consistent across time. However, none of these studies were actually designed to test how consistent the visuohaptic and haptic-haptic matching errors are across time, and they did not really attempt to quantify the amount of consistency. In the present study, we used a new measure to quantify the consistency of matching errors over time intervals varying from 5 min up to a month. Subjects had to reach with the index finger of their dominant hand towards visual or haptic targets (a point projected on a surface or the index finger of their non-dominant hand). The consistency of the matching errors over time was explored and quantified by reproducing this task 5 times within a 2 -month period.

\section{Methods}

\subsection{Subjects}

Ten subjects (one left-handed, 3 men, 26-39 years of age) took part in the experiment, including one of the authors (IK). The experiment is part of an ongoing research program that has been approved by the ethics committee of the Faculty of Human Movement Sciences of VU University Amsterdam. All subjects gave their written informed consent.

\subsection{Set-up and apparatus}

We used the same mirror set-up as we used previously to test visuohaptic and haptic-haptic matching errors in different experimental designs (Kuling et al., 2014) (Fig. 1A). In this set-up visual targets (dots, $15 \mathrm{~mm}$ diameter) could be projected onto a horizontal surface above a (semi-silvered) mirror. We used 6 different target positions (as in Kuling et al., 2014). A thin board ( $5 \mathrm{~mm}$ ) was placed at the same height as the apparent height of the projection plane as seen through the

A

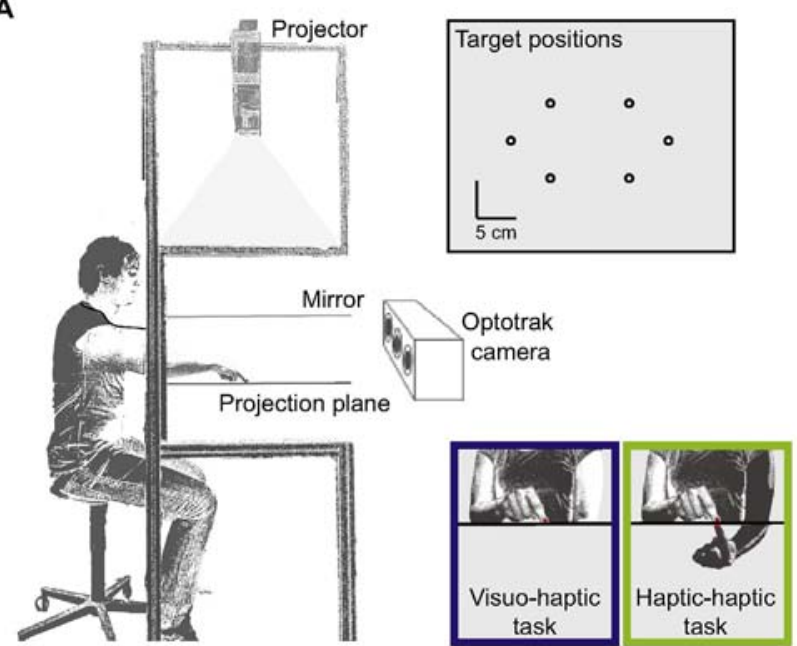

mirror. During the experiment, the subject could not see his or her arms and hands because they were looking at the screen above the mirror.

Position data were recorded with an Optotrak 3020 system (Northern Digital, Waterloo, Canada) at a sampling rate of $200 \mathrm{~Hz}$. Two infrared emitting diodes were placed on the nail of the left and right index finger to record the movements. Before each block of trials, the Optotrak coordinates of the dominant index finger were aligned with the coordinates of the visual presentations (projector coordinates). During this calibration we turned on the light below the semi-silvered mirror so that both the finger and target were visible.

We used two tasks. In the first task subjects attempted to match the index finger of their unseen dominant hand with a visually displayed target (visuo-haptic matching task). In the second task, the index finger of the subjects' non-dominant hand was guided towards a target location below the board, and the subjects attempted to match the location with the index finger of their dominant hand (haptic-haptic matching task).

\subsection{Procedure}

There were five sessions, each consisting of 4 blocks, alternating between the two tasks. Half the subjects started with a block of the visuo-haptic matching task, and the other half with a block of the haptic-haptic matching task. Within each block, the six different target positions were presented ten times in semi-random sequences (making sure that the first position was never the same as the last position of the previous sequence), which resulted in 60 trials per block. Each session took about 20 min per subject. There were fixed time intervals between the sessions. Each interval was (almost) 5 times larger than the previous one, starting with an interval of 5 h, then 24 h, 5 days, and 24 days.

The subjects received verbal instructions about the task in each block. They were instructed to reach for the target in one accurate movement and to stop at the position of the target. In the visualhaptic matching task, subjects had to move their unseen dominant index finger above the board towards the visually presented dot. The next dot then appeared and the subject reached for it, and so on. In the haptic-haptic matching task, twenty identical arrows were presented on the screen, representing the vector from the unseen, nondominant index finger below the board to the invisible target location. These arrows guided the non-dominant index finger to the target

B

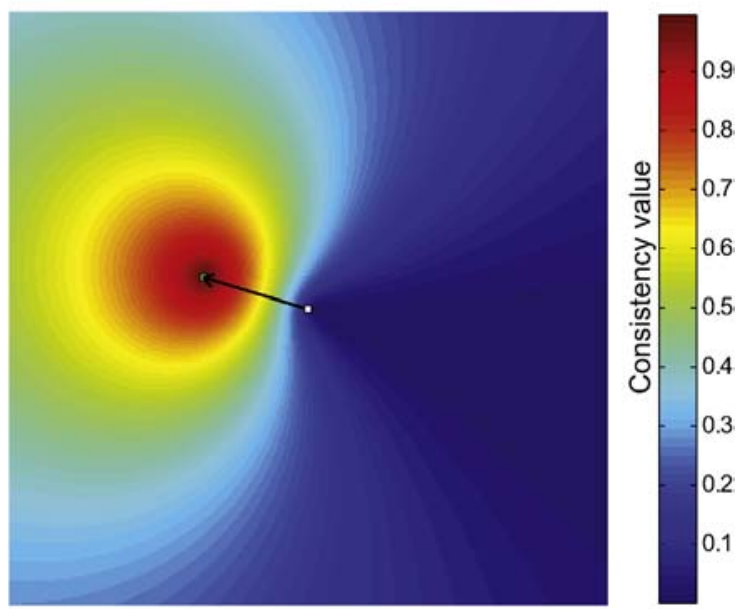

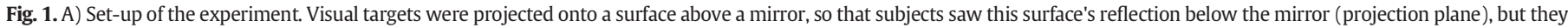

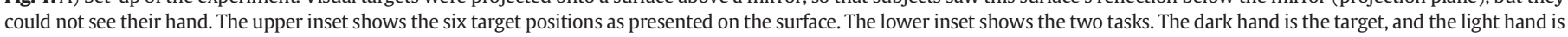

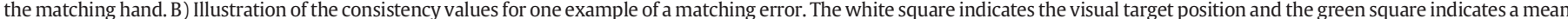

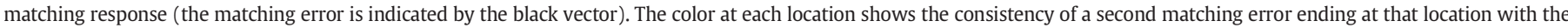

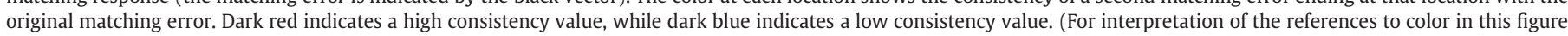
legend, the reader is referred to the web version of this article.) 
location for that trial. The length of the arrows was one tenth of the distance between the index finger and the target. Once the finger was at the position of the target (within $2 \mathrm{~mm}$ ), the arrows disappeared, and the subject had to move their unseen dominant index finger above the board to match the position of the other index finger. This method is similar to the arrow method used by Sabes and colleagues (Sober \& Sabes, 2005; Cheng \& Sabes, 2007).

\subsection{Analysis}

The endpoint of a finger movement was defined as the last of 8 successive frames for which the marker was moving slower than $3.5 \mathrm{~cm} / \mathrm{s}$ after having reached a velocity of $50 \mathrm{~cm} / \mathrm{s}$. For each subject, block, target $(\mathrm{t})$ and trial (i) we calculated the matching error $(M E)$, which is the vector between the target $(T)$ and the endpoint of the finger movement towards this target $(X)$.

$M E_{t, i}=X_{i}-T_{t}$.

Furthermore, for each subject, block and target we calculated, as a measure of variability, the area of the $95 \%$ confidence ellipse of the distribution of endpoints (this is the smallest possible ellipse that contains approximately $95 \%$ of the underlying data, assuming that the points are normally distributed in all directions). One of the subjects missed the first session in the schedule, so for this subject both the data of the first session and the comparison between the first and second session is missing.

For both the magnitude of the matching error and the variability (the area of the ellipse), a $2 \times 5 \times 2 \times 6$ repeated measures ANOVA (task $\mathrm{x}$ session $x$ block $x$ position) was used to determine whether matching errors and variability differed between tasks, sessions, blocks and positions. Greenhouse-Geisser corrections were used when sphericity was violated.

\subsubsection{Consistency value}

Because we are interested in the consistency of the matching errors over time, we developed a new measure of consistency between two matching errors $\left(M E_{1}\right.$ and $\left.M E_{2}\right)$ : the consistency value CONS. It incorporates both the magnitude and the direction of the matching errors. It is defined as

$\operatorname{CONS}\left(M E_{1}, M E_{2}\right)=1-\frac{\left|M E_{1}-M E_{2}\right|}{\left|M E_{1}\right|+\left|M E_{2}\right|}$

with $M E_{1}$ and $M E_{2}$ vectors as defined in Eq. (1). This consistency measure can be used for all error-pairings, such as for two matching errors of two single trials for a single target, for mean matching errors of two subjects, and for mean matching errors of the same subject for two different targets.

A consistency value of 1 means that the two matching errors are exactly the same (same direction and same magnitude), whereas a value of 0 means that they are in exactly opposite directions (irrespective of their magnitudes). A consistency value of 0.5 means that the magnitude of the difference vector between the two matching errors is the same as the mean magnitude of these matching errors (i.e. the errors differ from each other as much as they do from the target). How the consistency value for a second error varies over space with respect to a given error vector is shown in Fig. 1B.

The consistency value was calculated separately for each subject's matching errors $\left(\mathrm{CONS}_{\text {individual }}\right)$ based on the mean matching error for two series of trials:.

$\mathrm{CONS}_{\text {individual }}=\frac{1}{6} \sum_{t=1}^{6} \operatorname{CONS}\left(\overline{\mathrm{ME}}_{t}^{\text {series } 1}, \overline{\mathrm{ME}}_{t}^{\text {series } 2}\right)$

This was done separately for comparing the two blocks of the same task within each session (series 1 and series 2 correspond then to the two blocks), and for comparing the same task in all pairs of successive sessions as well as for comparing the first with the last session (series 1 and series 2 correspond then to the two sessions) The consistency value was calculated between the means of the matching errors for one target in the two blocks of a single session (within sessions) or between the means of the matching errors for one target in two sessions (between sessions). The consistency values were then averaged across the different target positions to give a single value per participant.

Although the value of CONS can theoretically vary between 0 and 1 , these values will never be found. The value of one will never be obtained because subjects make variable errors in addition to the (systematic) matching errors. The value of zero will never be obtained because if subjects are totally inconsistent, the second matching error will be random, not exactly in the opposite direction than the initial matching error.

To relate the consistency between blocks and sessions to the variability within these blocks and sessions (i.e. to a value that one could expect if the variations between sessions were equal to the variability within blocks of trials), we also determined a consistency value based on the variability between trials within each block. This measure, $\mathrm{CONS}_{\mathrm{var}}$, is defined as the mean of the consistency values between the single trials for a target within a block. It was then averaged across targets.

$\mathrm{CONS}_{\text {var }}=\frac{1}{540} \sum_{t=1}^{6} \sum_{i=1}^{10} \sum_{j=1}^{10} \operatorname{CONS}\left(\mathrm{ME}_{t, i}, M E_{t, j \neq i}\right)$

$\mathrm{CONS}_{\mathrm{var}}$ provides an estimate of the value that one would expect for $\mathrm{CONS}_{\text {individual }}$ if the variability across blocks and sessions were similar to that across trials within a single block, meaning that participants' matching errors are consistent across time. The indices $i$ and $j$ refer to the ten individual trials, so this measure compares all possible pairs of trials for a specific target within a specific block. To get a value of $\mathrm{CONS}_{\mathrm{var}}$ for each comparison that we want to make, we average across the relevant blocks and sessions.

To estimate a realistic minimal individual consistency value that considers any biases that are consistent across participants, we also determined the consistency between subjects. This measure, $\mathrm{CONS}_{\text {rand }}$, was determined for each subject by calculating the average consistency value of the subject's matching errors with all matching errors for the same task performed by other subjects.

$\mathrm{CONS}_{\text {rand }}=\frac{1}{540} \sum_{t=1}^{6} \sum_{k=1}^{10} \sum_{p=1}^{10} \operatorname{CONS}\left(\overline{M E}_{t, k}, \overline{M E}_{t, p \neq k}\right)$.

The indices $k$ and $p$ represent the 10 subjects, so the comparison is between the mean data of one subject for a specific target and the mean data of each of the other subjects for the same target. We averaged the $\mathrm{CONS}_{\text {rand }}$ for the within and between session comparisons in the same way as we did for $\mathrm{CONS}_{\mathrm{var}}$ The different CONS measures are listed in Table 1.

A $2 \times 5 \times 3$ repeated measures ANOVA (task $\times$ session $\times \mathrm{CONS}_{\mathrm{x}}$ ) was used to determine whether the consistency values between blocks within a session differed between tasks and sessions, and how the individual consistency values related to $\mathrm{CONS}_{\text {rand }}$ and $\mathrm{CONS}_{\mathrm{var}}$. A second $2 \times 5 \times 3$ repeated measures ANOVA (task $\times$ interval $\times \mathrm{CONS}_{\mathrm{x}}$ ) was used to see whether the consistency values differed between the sessions. For the latter analysis, we considered five different time intervals: four were comparisons between subsequent sessions, and the last was the mean of the five within-session comparisons (as a measure of the comparison across a short time interval). In both analyses, the effects in which we are specifically interested are the main effects of block or session, and whether the individual consistency values differ from $\mathrm{CONS}_{\text {rand }}$ and $\mathrm{CONS}_{\mathrm{var}}$. If the matching errors do not change with time, $\mathrm{CONS}_{\text {individual }}$ should be larger than $\mathrm{CONS}_{\text {rand }}$ and similar to (or larger than) $\mathrm{CONS}_{\mathrm{var}}$. 
Table 1

Overview of the different consistency values and their definitions.

\begin{tabular}{|c|c|}
\hline $\mathrm{CONS}_{\mathrm{x}}$ & Explanation \\
\hline $\mathrm{CONS}_{\text {individual }}$ & $\begin{array}{l}\text { The consistency of a subject's matching errors across blocks } \\
\text { or sessions }\end{array}$ \\
\hline $\mathrm{CONS}_{\mathrm{var}}$ & $\begin{array}{l}\text { An estimate of consistency based on the variability within a } \\
\text { single block }\end{array}$ \\
\hline $\mathrm{CONS}_{\text {rand }}$ & $\begin{array}{l}\text { The minimal expected value based on the consistency } \\
\text { between subjects }\end{array}$ \\
\hline
\end{tabular}

\section{Results}

The mean errors of one subject for all blocks of all 5 sessions (Fig. 2) show large differences between the matching errors for the two tasks (blue and green clearly separated), and small differences between blocks and sessions of the same task (same colored symbols are similar to each other).

The $2 \times 5 \times 2 \times 6$ repeated measures ANOVA on the magnitude of the mean matching errors for each target showed main effects of block and target position (block $F_{1.0,8.0}=17.7, p=0.003$, position $\mathrm{F}_{5,40}=2.6$, $p=0.039$ ). There was no significant effect of task or session (task $F_{1.0,8.0}=0.0, p=0.86$; session $F_{4,32}=1.9, p=0.14$ ), and no significant interaction (Fig. 3A). The magnitude of the mean matching error was significantly larger in the second block of each session (increasing from $3.0 \mathrm{~cm}$ to $3.3 \mathrm{~cm}$ ). It is unclear why this was found. The effect of target position corresponded with a smaller magnitude of the matching error for targets closer to the body $(2.9 \mathrm{~cm}$ for the targets closest to the body and $3.4 \mathrm{~cm}$ for the furthest targets), which is line with previous research (Van Beers et al., 1998; Kuling et al., 2014).

The $2 \times 5 \times 2 \times 6$ repeated measures ANOVA on the variability showed a significant effect of position $\left(F_{5,40}=4.9, p=0.001\right)$ and a significant task by block interaction $\left(F_{1.0}, 8.0=9.3, p=0.016\right)$. There was no effect of task, session or block (task $F_{1.0,8.0}=1.1, p=0.32$; session $\mathrm{F}_{2.1,17.0}=0.7, p=0.54$, block $F_{1,8}=0.1, p=0.72$, Fig. 3B). The variability was higher for targets further from the body $\left(14.5 \mathrm{~cm}^{2}\right.$ for the targets closest to the body and $18.5 \mathrm{~cm}^{2}$ for the furthest targets), which is in line with previous research (Van Beers et al., 1998; Kuling et al., 2014). The interaction effect is caused by the variability decreasing in successive

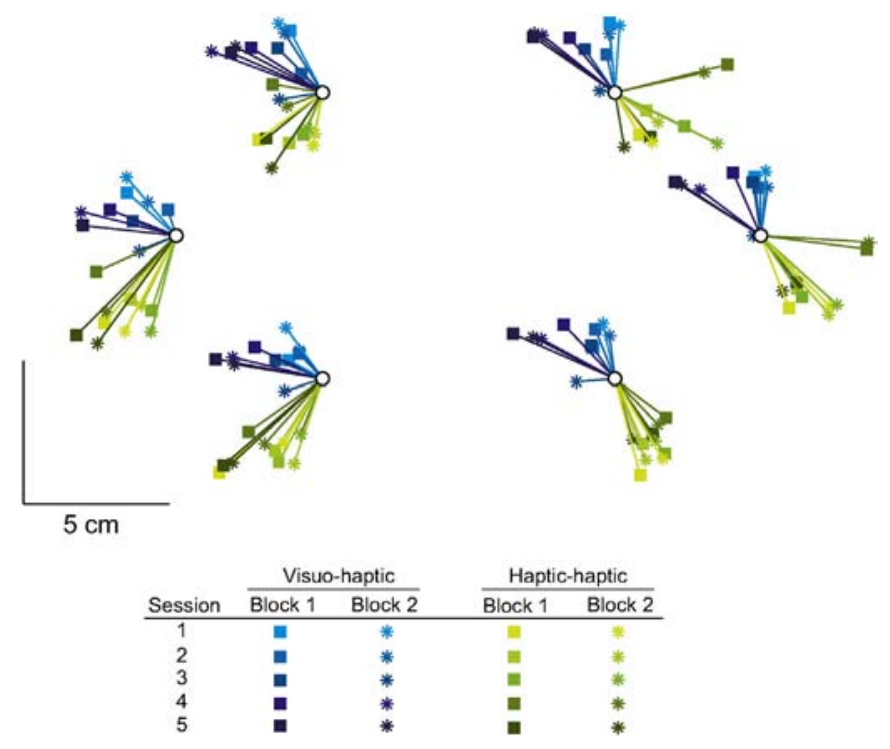

Fig. 2. Mean error for each block of one subject. The white dots show the target positions. The arrows show the mean matching errors for the different blocks and sessions. Each session is represented by a slightly different color; the lightest colored symbols show the matching errors in the first session, while the darkest colors show the matching errors in the last session. Visuo-haptic and haptic-haptic matching errors are clearly different, but within one task there is considerable consistency. blocks for the haptic-haptic task while increasing slightly for the visual-haptic task.

Within each session, the consistency between individual subjects'

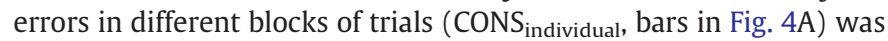
much higher than the consistency between different subjects' errors $\left(\mathrm{CONS}_{\text {rand, }}\right.$, white symbols in Fig. $\left.4 \mathrm{~A}\right)$. The $\mathrm{CONS}_{\text {rand }}$ is on average about 0.3. In Fig. 1B, this corresponds to the cyan region; indicating matching errors that are perpendicular to the original matching error, which seems in line with a random second error. The $2 \times 5 \times 3$ repeated measures ANOVA on the consistency values showed only a significant main effect of $\mathrm{CONS}_{\mathrm{x}}\left(\right.$ task $F_{1.0,8.0}=0.48, p=0.508$, session $F_{4,32}=$ $\left.2.37, p=0.074, \mathrm{CONS}_{\mathrm{x}} \mathrm{F}_{2,16}=316.8, p<0.001\right)$ and a significant interaction between $\operatorname{CONS}_{\mathrm{x}}$ and task $\left(\mathrm{F}_{2,16}=3.74, p=0.046\right)$. Post-hoc comparisons with Bonferroni correction showed that the $\mathrm{CONS}_{\text {individual }}$ were larger than both $\mathrm{CONS}_{\text {rand }}(p<0.001)$ and $\mathrm{CONS}_{\mathrm{var}}(p=0.005)$. The interaction indicates that $\mathrm{CONS}_{\text {individual }}$ was larger for haptichaptic matching than visuo-haptic matching, whereas the other two consistency measures are independent of the task.

Individual subjects' matching errors were quite consistent across successive sessions (the four pairs of bars in the middle in Fig. 4B). As expected the consistency for random pairings of errors by different subjects was the same between and within session (white symbols are at the same level in Fig. 4A and B). The last pair of bars in Fig. 4B (marked $\mathrm{S} 1-\mathrm{S} 5$ ) is just as high as the other pairs, which means that the consistency of the matching errors between the first and the last session hardly differs from the consistency for the comparisons between the four successive sessions. This indicates that the small inconsistencies between sessions were not due to a systematic drift across sessions. In the statistical analyses, comparisons over five different time intervals were used; four comparisons between subsequent sessions, and one for the mean of the five within-session comparisons. The $2 \times 5 \times 3$ repeated measures ANOVA on the consistency values showed again only a significant main effect of $\mathrm{CONS}_{\mathrm{x}}$ (task $F_{1.0,8.0}=0.191, p=0.674$, session $F_{4,32}=1.74$, $\left.p=0.165, \mathrm{CONS}_{\mathrm{x}} \mathrm{F}_{2,16}=229.2, p<0.001\right)$ and no significant interactions. Post-hoc comparisons with Bonferroni correction showed that the $\mathrm{CONS}_{\text {individual }}$ were larger than the $\mathrm{CONS}_{\text {rand }}(p<0.001)$, and did not differ from $\mathrm{CONS}_{\mathrm{var}}(p=0.626)$.

\section{Discussion}

In this study we investigated the consistency of visuo-haptic and haptic-haptic matching errors over time intervals from several minutes up to a month. The results confirm that the matching errors are subjectdependent (Van Beers et al., 1998; Smeets et al., 2006; Sousa et al., 2010; Rincon-Gonzalez et al., 2011; Kuling et al., 2013; Van der Kooij et al., 2013; Kuling et al., 2014), and show that they are also very consistent across time. Previously, Rincon-Gonzalez et al. (2011) found consistent visuo-haptic matching errors in a passive task, and Smeets et al. (2006) reported very consistent matching errors in an active task. We extended their tasks by adding multiple time intervals and looking at the matching errors for both visuo-haptic and haptic-haptic tasks. The consistency of the matching errors between sessions did not differ from the consistency between blocks within a session.

To quantify the consistency, we came up with a value that takes into account both the magnitude and the direction of matching errors, irrespective of the variability. To relate this consistency value to the variability within the visuo-haptic and haptic-haptic matching tasks, we also estimated a consistency value on the basis of the variability within each block. The consistency of both visuo-haptic and haptic-haptic matching errors is of the same order of magnitude as the variability within a block of trials (Fig. 4). For short time intervals (within sessions) the consistency is even larger than this estimate based on the variability, presumably because the former measure is based on averaged data.

Although we now know that the visuo-haptic and haptic-haptic matching errors are very consistent across long periods of time, their origin is still unknown. One possible explanation is that there are sensory 
A

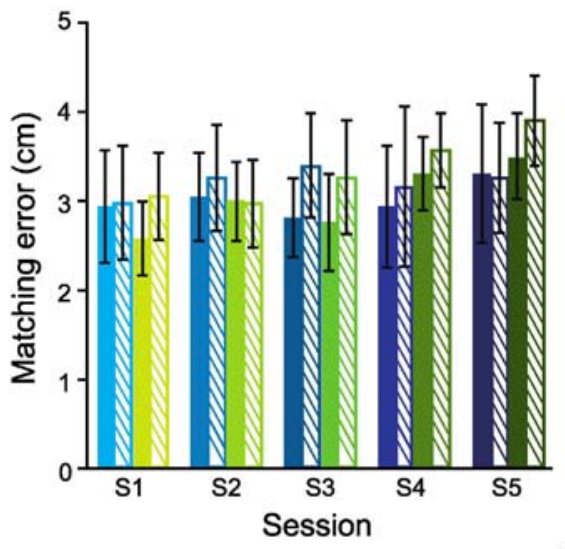

B

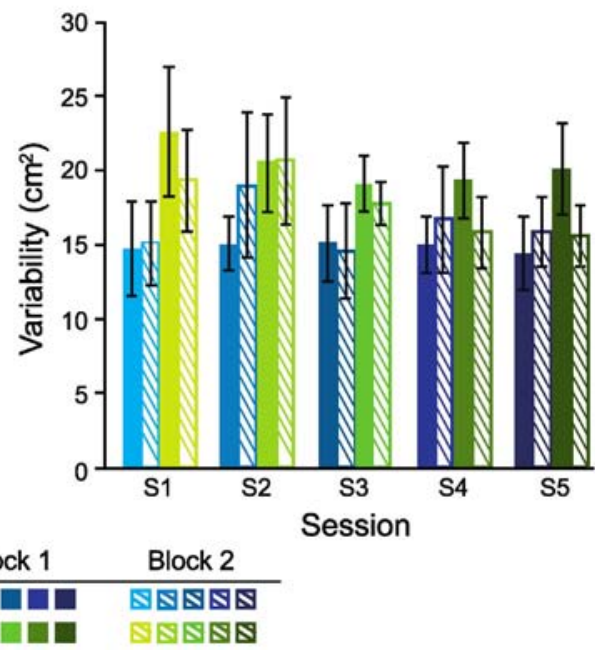

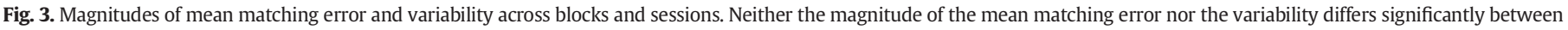

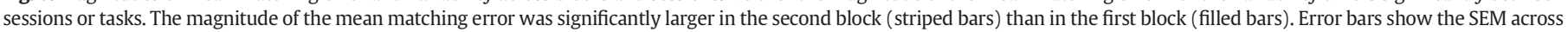
subjects.

mismatches due to inherent or induced biases in one or more modalities. Bernier et al. (2007) showed that adapting to prisms during movements to visual targets did not influence subsequent movements to haptic targets. They interpreted this as evidence that there are different spatial maps for different sensory modalities, each of which is adjusted separately on the basis of experience, and therefore need not be aligned with other maps. Smeets et al. (2006) used a similar idea of the presence of separate inherently mismatching visual and proprioceptive estimates to explain the drift in pointing movements after removing visual feedback. When there is no visual feedback of the moving hand, the errors that participants make drift towards the inherent visuo-haptic error. During normal daily functioning, subjects use the combined visuohaptic estimate for the location of both the hand and the target. Consequently, performance is without problems, so there is no reason for adapting the mapping.

Another possible explanation for the matching errors is that there are systematic errors in the transformation of the information between modalities. McIntyre and Lipshits (2008) found patterns of errors in orientation-matching in a visuo-haptic task that were not present in separate visual and haptic tasks, and therefore could not be explained by biases in the separate senses. They attributed these errors to the sensorimotor transformations between the visual and haptic sensory systems. Here too, it is unclear why such errors persist despite the constant feedback that is provided in daily life. Further research is required to determine to what extent the matching error finds its origin in sensory errors in the separate modalities or in the transformation between the two modalities.

\section{Conclusion}

In this study we investigated the consistency of visuo-haptic and haptic-haptic matching errors over time. To do so we developed a measure of consistency in which both the direction and the magnitude of the matching error is taken into account. Overall, we show that visuohaptic and haptic-haptic matching errors are very consistent over
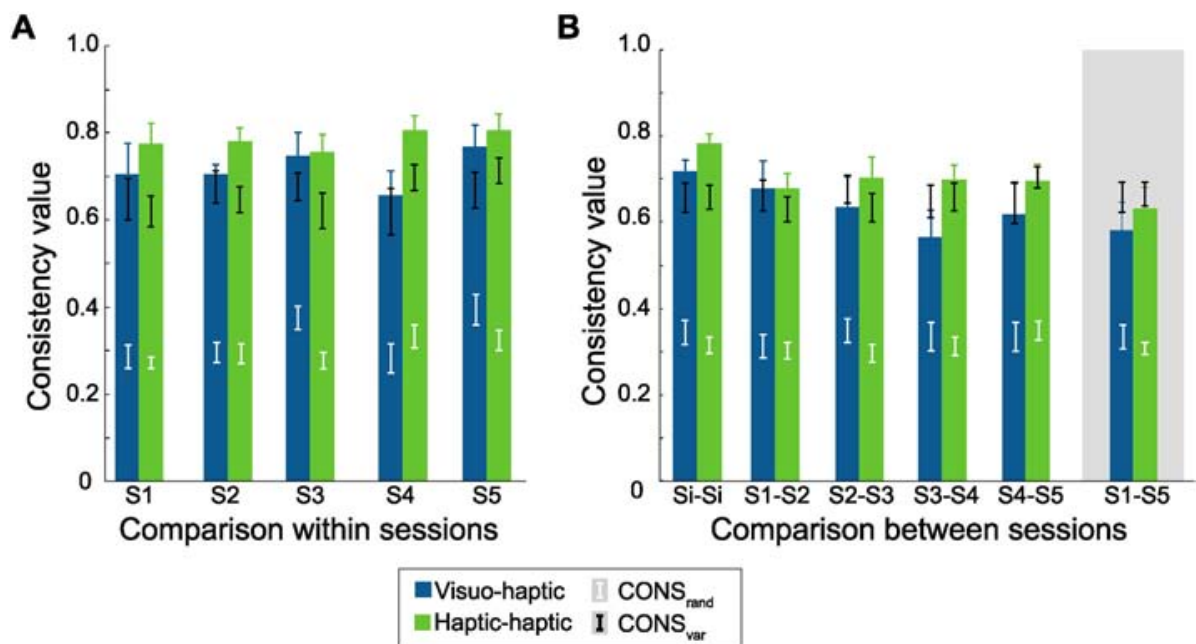

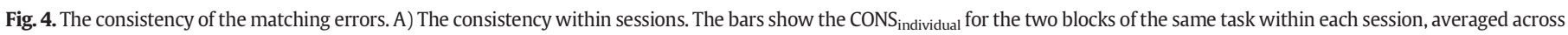

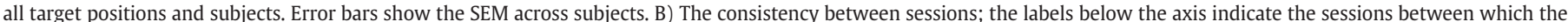

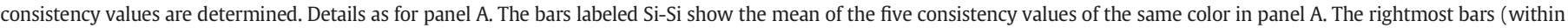

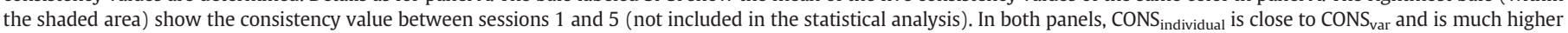
than $\mathrm{CONS}_{\text {rand. }}$ 
time. The consistency is of the same order of magnitude as the variability within single visuo-haptic and haptic-haptic matching tasks.

\section{Acknowledgments}

This research is part of the H-Haptics perspective program, supported by the Dutch Technology Foundation STW, which is part of the Netherlands Organization for Scientific Research (NWO) and partly funded by the Ministry of Economic Affairs, Agriculture and Innovation. STW grant 12160.

\section{References}

van Beek, F. E., Tiest, W. M. B., \& Kappers, A. M. (2013). Anisotropy in the haptic perception of force direction and magnitude. IEEE Transactions on Haptics, 6, 399-407.

van Beers, R. J. (2009). Motor learning is optimally tuned to the properties of motor noise. Neuron, 63, 406-417.

Bernier, P. -M., Gauthier, G. M., \& Blouin, J. (2007). Evidence for distinct, differentially adaptable sensorimotor transformations for reaches to visual and proprioceptive targets. Journal of Neurophysiology, 98, 1815-1819.

Cheng, S., \& Sabes, P. N. (2007). Calibration of visually guided reaching is driven by errorcorrective learning and internal dynamics. Journal of Neurophysiology, 97, 3057-3069.

Haggard, P., Newman, C., Blundell, J., \& Andrew, H. (2000). The perceived position of the hand in space. Perception \&' Psychophysics, 68, 363-377.

Kuling, I. A., Brenner, E., \& Smeets, J. B. J. (2013). Proprioception is robust under external forces. PloS One, 8, e74236.

Kuling, I. A., Brenner, E., \& Smeets, J. B. J. (2015). Torques do not influence proprioceptive localization of the hand. Experimental Brain Research, 233, 61-68.
Kuling, I. A., Brenner, E., \& Smeets, J. B. J. (2016). Proprioceptive localization of the hand changes when skin stretch around the elbow is manipulated. (submitted for publication).

Kuling, I. A., van der Graaff, M. C. W., Brenner, E., \& Smeets, J. B. J. (2014). Proprioceptive biases in different experimental designs. In M. Auvray, \& C. Duriez (Eds.), Haptics: Neuroscience, Devices, Modeling, and Applications (pp. 18-24). Berlin Heidelberg: Springer.

McIntyre, J., \& Lipshits, M. (2008). Central processes amplify and transform anisotropies of the visual system in a test of visual-haptic coordination. Journal of Neuroscience, 28, 1246-1261.

Rincon-Gonzalez, L., Buneo, C. A., \& Helms Tillery, S. I. (2011). The proprioceptive map of the arm is systematic and stable, but idiosyncratic. PloS One, 6, e25214.

Smeets, J. B. J., van den Dobbelsteen, J. J., de Grave, D. D., van Beers, R. J., \& Brenner, E. (2006). Sensory integration does not lead to sensory calibration. Proceedings of the National Academy of Sciences of the USA, 103, 18781-18786.

Sober, S. J., \& Sabes, P. N. (2005). Flexible strategies for sensory integration during motor planning. Nature Neuroscience, 8, 490-497.

Sousa, R., Brenner, E., \& Smeets, J. B. J. (2010). A new binocular cue for absolute distance: disparity relative to the most distant structure. Vision Research, 50, 1786-1792.

Van Beers, R. J., Sittig, A. C., \& Denier van der Gon, J. J. (1998). The precision of proprioceptive position sense. Experimental Brain Research, 122, 367-377.

Van der Kooij, K., Brenner, E., van Beers, R. J., Schot, W. D., \& Smeets, J. B. J. (2013). Alignment to natural and imposed mismatches between the senses. Journal of Neurophysiology, 109, 1890-1899.

Von Hofsten, C., \& Rösblad, B. (1988). The integration of sensory information in the development of precise manual pointing. Neuropsychologia, 26, 805-821.

Wong, T. S. (1977). Dynamic properties of radial and tangential movements as determinants of the haptic horizontal-vertical illusion with an ' $L$ ' figure. Journal of Experimental Psychology: Human Perception and Performance, 3, 151-164. 